\title{
Schreiben des Herrn Prof. Wolf an den Herausgeber.
}

Die soeben erschienene Nr. 40 meiner ,Astronomischen Mittheilungen " giebt zum Abschlusse des vierten Bandes derselben kurze biographische Notizen über meinen langjährigen Correspondenten $\mathrm{H}$ ofrath Heinrich Schwabe von Dessau, für alle Zeiten berühmt durch seine erste Entdeckung der von mir nachher auf $11 \frac{1}{9}$ Jahr mittlerer Dauer bestimmten Sonnenperiode, - und meinen Jugendfreund Staatsrath Gottfried Schweizer in Moskau, bekannt durch seine Cometenentdeckungen, seine Bestinmung der sonderbaren Local- attractionen in der Nähe ron Moskau, etc. Im Weitern enthält sie eine Fortsetzung des räsonnirenden Verzeichnisses der Sammlungen der Züricher Steruwarte, und tritt namentlich einlässlich über das am Ende des 16. Jahrlıunderts von Joost Bürgi construirte Triangular-Instrument ein, von welchem die erwähnten Sammlungen ein von Bürgi's Gehülfen Heinrich Stolle construirtes Exemplar besitzen.

Zürich, den 6. August 1876.
R. Wolf.

\section{Beobacbtungen des Planeten (165).}

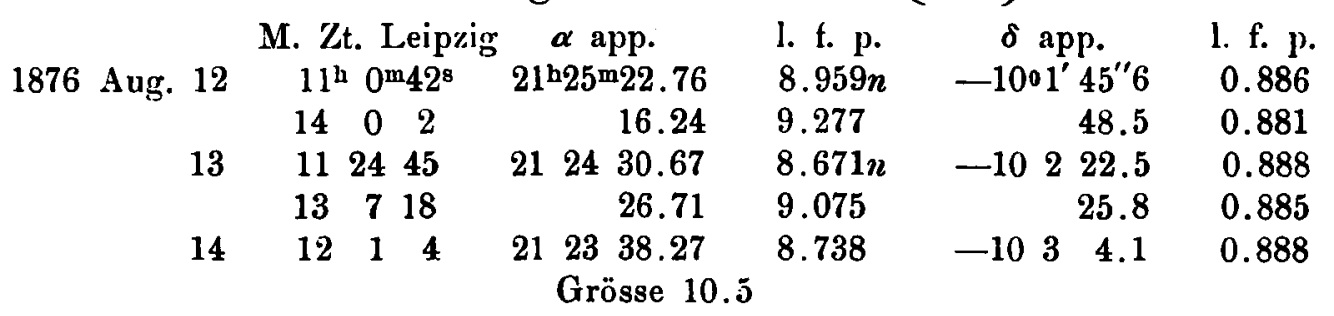

C. Bruhns.

\section{Ueber ein kleines Passagen-Instrument von A. Steger jun. in Kiel.}

In beiliegender Figurentafel ist in natürlicher Grösse die Zeichnung eines kleinen Passagen-Instrumentes gegeben, welches von dem Mechaniker A. Steger jun. hierselbst kïrzlich in mehreren Exemplaren angefertigt ist. Im dritten Bande der „Zeitschrift für pop. Mitth. aus dem Gebiete der Astromomie etc." habe ich bereits eine Beschreibung eines sulchen Instrumentes gegeben, und hat dieselbe wesentlich Herrn Steger bei der Ausführung zum Muster gedient. Als Abänderungen sind zu erwähnen erstens das Objectiv-Prisma, welches mit ebenen, nicht wie früher vorgeschlagen, mit convexen Kathetenflächen versehen ist, während das Objectiv aus einer achromatischen Linse besteht, und zweitens die Vorrichtung zur Beleuchtung der Fäden, die hier durch einen von einer kleinen Lampe beleuchteten, auf der Hypotenusenfläche des Prismas eingeschliffenen matten Ring bewirkt wird.

\section{Entdeckung eines Planeten (166).}

Depesche aus Washington: Planet (166) entdeckt ron Professor Peters in Clinton. 1876 A ug. $10 \quad \alpha 21^{\text {h } 30^{\mathrm{m}}} \delta-19^{\circ} 23^{\prime} \quad$ Bew. $-10^{\prime} \quad$ Grösse 11.

In halt:

Zu Nr. 2103. S. W. Bumham. Seventh Calalogue of New Double Stars. 225. - J. F. Julius Schmidt. Verănderliche Sterne. Allıen 1876. 229. - W. Doberck. New Elements of 6 Coronae Borealis. 233. - Hugo Gericke. Kreismicrometer-Beobachtungen kleincr Planeten. 237. - Wolf. Schreiben an den Herausgeber. 239. - C. Bruhns. Beobachtungen des Planeten (165). 239. $-C$. $F$. W. Peters. Ueber ein kleines Passagen-Instrument von A. Steger jun. in Kiel. 239. - Entdeckung eines Planeten (166). 239.

Druck von Fiencke \& Schachel in Kiel. - Kiel. 1876, August 31. 